\title{
Diseño de un sistema para la gestión de inventarios de las pymes en el sector alimentario
}

Diego Andrés CARreño Dueñas ${ }^{1}$ Luis Felipe Amaya GonzÁlez ${ }^{2}$

ERIKA TATIANA Ruiz ORJUELA ${ }^{3}$ FeLIPE JAVIER TiBOCHE ${ }^{4}$

ReCIBIDO: 05/12/2018 AcePTADo: 15/02/2019

\begin{abstract}
RESUMEN
En el presente artículo se ha diseñado una propuesta para mejorar el sistema en torno a la gestión de inventarios de las pymes, sobre todo, las del sector productor y comercializador de dulces. El objetivo de la investigación es aumentar la productividad del manejo y control de inventarios; por ello, la metodología consiste en realizar una revisión bibliográfica acerca del contexto y los retos que presentan las pymes con la aplicación de las TIC. Luego, el diagnóstico de la empresa estudiada permitirá calcular el pronóstico basado en el procedimiento más acertado identificado en la fase anterior. En conclusión, lo que se busca es implementar dicho sistema de control de inventarios, lo cual se logrará mediante el uso de un software diseñado especialmente para esta organización, el cual utilizará códigos QR para la suministración y actualización de datos en tiempo real.

Palabras-claves: Cantidad económica de pedido; código QR; control de inventarios; gestión de inventarios; sistema de inventarios.
\end{abstract}

\section{INTRODUCCIÓN}

Principalmente, se debe señalar que el inventario es una parte fundamental dentro de las operaciones de una organización, pues este lleva a cabo el registro de las actividades productivas y comerciales. Por ello, debe presentarse en un estado que impida el sobreabastecimiento de productos que le cueste una gran suma de dinero a la empresa; al mismo tiempo, el gestor debe calcular los pedidos a través del inventario para que no se hallen faltantes. Por ese motivo, Hillier y Lieberman (2010) mencionan que "[...] los inventarios prevalecen en el mundo de los negocios. Mantenerlos en un buen nivel es necesario para las compañías que operan con productos físicos, como fabricantes, distribuidores y comerciantes" (p. 772).

En Colombia, las cifras aproximadas indican que las pymes:

[...] constituyen más del $90 \%$ de las entidades económicas, el $75 \%$ de los nuevos puestos de empleo y el $53 \%$ de la producción bruta de los sectores industrial, comercial y de servicios se conviert[e]n en parte de la esencia de la economía nacional, no sólo por el volumen de este tipo de empresas, sino también por la relevancia que tienen en la contribución a los índices de empleo del país. (Quintero, 2018, p. 12 )

De esta forma, datos como los proporcionados por la Gran Encuesta a las Microempresas, elaborada por la Asociación Nacional de Instituciones Financieras (2018), revelan los problemas más comunes que enfrentan estas pequeñas y medianas empresas. Algunas de ellas han sido evidenciadas también en el estudio de Regalado (2007), donde se menciona que el "acceso restringido a las fuentes de financiamiento, bajos niveles de capacitación de sus recursos humanos, limitados niveles de innovación y de-

1 Magíster en Diseño y Gestión de Procesos y docente investigador de la Universidad Pedagógica y Tecnológica de Colombia, Boyacá, Colombia.

E-mail: diego.carreno@uptc.edu.co

2 Especialista en Ingeniería de Producción y Operaciones y docente investigador de la Universidad Pedagógica y Tecnológica de Colombia. Boyacá, Colombia.

E-mail: luisfelipe.amaya@uptc.edu.co

3 Magíster en Ingeniería Industrial y docente investigador de la Universidad Pedagógica y Tecnológica de Colombia. Boyacá, Colombia. E-mail: erika.ruiz03@uptc.edu.co

4 Administrador industrial e investigador en la Universidad Pedagógica y Tecnológica de Colombia. Boyacá, Colombia.

E-mail: felipe.tiboche@uptc.edu.co 
sarrollo tecnológico, baja penetración en mercados internacionales, bajos niveles de productividad, baja capacidad de asociación y administrativa" (p. 73).

Asimismo, investigaciones anteriores evidencian problemas que han permanecido en el tiempo, como lo muestra el estudio de Gutiérrez y Rodríguez (2008), el cual concluye que las empresas pymes en Colombia no llevan un control de ventas y pérdidas que permita medir el nivel del servicio prestado con el fin de determinar el nivel óptimo de inventario de cada producto terminado. Se analizaba también el hecho de que la mayoría de empresas tome decisiones con base en la experiencia y en modelos de gestión apoyados en técnicas cualitativas; y, por otro lado, en menor proporción con base en métodos estadísticos de pronóstico de demanda. Además, se encontró que el $10 \%$ de las empresas que implementaron algún tipo de tecnología para ayudar al control del inventario de la organización tuvieron que adaptar sus procesos a los requisitos del software, cuando debería ocurrir al contrario.

Todos los inconvenientes mencionados en el párrafo anterior pueden ser solucionados —destaca Argüello (2015) en su diagnóstico de la gestión de inventarios de las pymes del sector industrial- a través de una eficiente gestión del inventario. De acuerdo con lo propuesto, se establece que las TIC juegan un papel clave en la gestión de inventarios, puesto que en el mercado existen diferentes herramientas informáticas destinadas al apoyo en la gestión de la cadena de suministro. Entre ellas podemos encontrar las siguientes: VMI (inventario administrado por el proveedor), CPFR (planeamiento participativo, pronóstico y reabastecimiento), el RFID (identificación por radiofrecuencia) y el código de barras, las cuales son utilizadas para la identificación, registro de operaciones y trazabilidad; y WMS (sistema de gestión de almacenes) y LMS (sistema de manejo de personal), las cuales apoyan la gestión de los flujos de información al ser realizados de forma correcta (Correa y Gómez, 2009).

Pese al apoyo que las herramientas informáticas brindan, las pymes colombianas argumentan que no implementan alguna TIC por desconocimiento o capacidad de inversión; mientras que aquellas que deciden realizar la implementación no cuentan con personas con conocimiento de las TIC y su grado de aplicación en las organizaciones (Correa, Álvarez y Gómez, 2010; Argüello, 2015).

Por otro lado, según analizan Servera, Gil y Fuentes (2009), las TIC juegan un papel clave en la calidad del proceso de gestión de inventarios de las empresas, pues demuestran en su investigación la relevancia de realizar un estudio sobre estas, aplicadas a la función logística y de gestión de materia prima. Así, la incorporación con rapidez de los avances en las TIC en la función logística es un factor diferencial que permite mejorar la eficiencia de todos los procesos porque genera valor al cliente.

Desde el punto de vista de Correa, Álvarez y Gómez (2010), el máximo grado de control del inventario se logra en las empresas con la implementación de sistemas automatizados de control e identificación de productos, como "las tecnologías de código de barras y la radiofrecuencia (RFID) [que] son sistemas de identificación de productos y captura de datos que contribuyen a que los flujos de información en la gestión de la cadena de suministro se realicen de manera eficiente y ágil" (pp. 117-118). No obstante, estos sistemas presentan problemas de almacenamiento y lecturas erróneas debido al nivel de madurez de la tecnología actual; por eso, Duong, Wood y Wang (2015) plantean dos opciones: utilizar estos dos sistemas de forma simultánea o buscar nuevas alternativas tecnológicas más rápidas, libres, ajustables y de menor costo tecnológico. A partir de ello, surge una opción novedosa de almacenamiento para gestión de inventarios: los códigos QR (respuesta rápida). Según Sandoval (2016), estos fueron creados por la empresa japonesa Denso Wave con fines logísticos y de control de inventario, pues "los códigos QR son un mobile tag, es decir, un conjunto de imágenes bidimensionales que se descodifican con un teléfono móvil y que permiten dar información más directa al usuario" (Vila, 2011, p. 1).

Si bien todas estas TIC deben estar soportadas bajo una metodología de control de inventarios, autores como Osorio (2008) recomiendan varios sistemas de gestión de inventarios para pymes, a los cuales los organiza en orden de dificultad e implementación: "control manual, sistema Two-Bin, revisión visual, puntos de reorden EOQ, puntos de reorden con lotes dependientes de inventario, revisión periódica con lotes dependientes del nivel de inventario y revisión periódica con puntos de reorden y lotes dependientes del nivel de inventario" (p. 6).

Para diseñar el sistema gestor de inventarios, en el caso de este estudio, se desarrolló un software capaz de calcular la cantidad económica de pedido, generar códigos QR y administrar la información en una base de datos. Con esta aplicación se evitarán los datos faltantes, se disminuirán los costos de almacenamiento y de personal, se ahorrará tiempo, se reducirán los niveles de inventario y se planeará 
la cantidad óptima de pedidos. Esto se logrará al tener al alcance del bolsillo un lector estándar con el cual actualizar en forma real la información de las existencias (Hillier y Lieberman, 2010).

\section{METODOLOGÍA}

En primer lugar, se elabora el cuestionario de entrevista dirigido al gerente, a la contadora y al supervisor de producción de la empresa con el fin de recolectar las variables más relevantes de la cadena de valor del inventario. Posteriormente, se tabulan los datos obtenidos en las entrevistas, de la misma manera como Hernández, Fernández y Baptista (2014) lo realizan. En último lugar, se verifica que el nivel de inventario que la empresa almacena no sea demasiado alto, pues se deben evitar pérdidas económicas por las fluctuaciones de la demanda. Así, lo más adecuado es que la empresa mantenga el stock del inventario en el nivel óptimo (Render y Heizer, 2004).

El sistema de gestión de inventarios debe ser diseñado de acuerdo con las necesidades de la empresa, ya que estas han sido identificadas en el diagnóstico inicial y se basan en las teorías de autores que han escrito sobre gestión de inventarios. De este modo, el inventario comprende las unidades de un bien como la materia prima, producto en proceso y producto terminado de la organización, y se encuentran disponibles para su uso en el almacén o en la planta de producción. En ese sentido, Chase, Jacobs y Aquilano (2009) definen al inventario como "las existencias de una pieza o recurso utilizado en una organización". Asimismo, señalan que "un sistema de inventario es el conjunto de políticas y controles que vigilan los niveles del inventario y determinan aquellos a mantener, el momento en que es necesario reabastecerlo y [saber] qué tan grandes deben ser los pedidos" (p. 547).

El pronóstico de demanda se calcula con el modelo de suavizamiento exponencial, que es la técnica de pronóstico más común y parte integral de casi todos los programas de pronóstico por computadora; por ello, se usan con mucha frecuencia al ordenar el inventario en empresas minoristas, compañías mayoristas y comercializadoras de productos perecederos. De esta forma, el estudio de Pérez, Mosquera y Bravo (2012) se sirve de esta práctica para comparar la utilidad entre los diferentes métodos de pronóstico en productos de consumo masivo en la canasta familiar. La conclusión a la que llegan es que el método de suavizamiento exponencial responde bien cuando la tendencia de ventas es creciente, tal y como se presenta en este caso de estudio.

La metodología de control de inventario seleccionada fue el modelo de cantidad económica de pedido (EOQ), escogida a partir del estudio de Osorio (2008), el cual recomienda que los modelos de gestión de inventarios se adecúen a las pymes colombianas. El modelo EOQ forma parte de la cuarta complejidad después de los modelos control manual, sistema Two-Bin y revisión visual, utilizados antes en la empresa analizada. Además, Piña (2012), al obtener buenos resultados en su investigación, recomienda este sistema de control en empresas con materias primas perecederas. El modelo EOQ floreció hacia el año de 1913 y fue uno de los modelos pioneros en el control de inventarios (Andriolo, Battini, Grubbström, Persona y Sgarbossa, 2014).

La ecuación (1), planteada por Hillier y Lieberman (2010), representa la fórmula EOQ, utilizada para calcular la cantidad óptima de productos a ordenar o producir por lote.

$$
Q=\sqrt{\frac{2 d K}{h}}
$$

Asimismo, la ecuación (2) corresponde al tiempo del ciclo.

$$
t=\sqrt{\frac{2 K}{d h}}
$$

Punto de reorden $=($ tasa de demanda $) \times($ tiempo de entrega)

Donde:

$\mathrm{K}=$ costo de preparación para ordenar un lote,

$\mathrm{d}=$ tasa de demanda, $\mathrm{y}$

$\mathrm{h}=$ costo de mantener el inventario por unidad de tiempo.

El diseño y la programación del software son procesos necesarios para la creación de sistemas de información dentro de las organizaciones, ya que permiten gestionar la información con mucha efectividad y administrar los datos de forma rápida, confiable, segura, en tiempo real y trazable. De acuerdo con Pressman (2010), "el software se ha incrustado profundamente en casi todos los aspectos de nuestras vidas" (p. 10); por lo que antes de desarrollar un software, es necesario entender plenamente el problema que se quiere solucionar. 
Para Quigley y Gargenta (2007), el software diseñado abarca las especificaciones y la estructura del sistema de gestión de inventarios requeridas por la organización, la cual se sostiene en la lectura de códigos QR para su alimentación y actualización de datos (Duong, Wood y Wang, 2015). Al ser una tecnología que puede almacenar más información que los códigos de barras, permite una mayor velocidad con los sistemas informáticos de la actualidad y cumple con las especificaciones requeridas para una pequeña empresa.

A partir de la afirmación de Pressman (2010), quien manifiesta que el software debe comprender un conjunto de programas escritos, se certifica que los lenguajes utilizados para programar el sistema de gestión de inventarios son PHP (preprocesador de hipertexto). Según Converse, Park y Morgan (2004), estos son un software libre, garantizado por un conjunto de esquemas de licencias que no tiene las restricciones de copyleft o copyright; es decir, que es un lenguaje de código abierto, como lo señalan Quigley y Gargenta (2007). Por otro lado, HTML (HyperText Markup Language) significa lenguaje de marcas de hipertexto, el cual usa un lenguaje de etiquetas para construir páginas web (Gauchat, 2012). Desde otro ángulo, CSS (Cascading Style Sheets) son hojas de estilo en cascada, que es un mecanismo simple para agregar estilos; por ejemplo, fuentes, colores o espaciado, a los documentos web (Quigley y Gargenta, 2007). Por otra parte, JavaScript $\circledast$ es un lenguaje interpretado, orientado a objetos de programación dinámico, que se utiliza en millones de páginas web y aplicaciones de servidor en todo el mundo (Valderrey, 2013). Por último, Quigley y Gargenta (2007) indican que el sistema gestor de bases de datos llamado MySQL es "la base de datos de código abierto más popular del mundo" (p. 7) [traducción propia]; pues es alimentado por un lector estándar de QR que parte de la innovación y optimización en costos y tiempo. Así, cualquier operario que posea un smartphone o iPhone podrá actualizar dicha información, con lo que se evitará el paro de producción en tareas de conteo y la actualización de inventarios manuales o por observación.

\section{RESULTADOS}

\section{Pronóstico de ventas del producto}

Por un lado, el método de suavizamiento logró una buena exactitud de pronóstico para el siguiente periodo, mientras que la gráfica de series de tiempo permitió indagar tendencias en los datos que se optiman a lo largo de un periodo (Morcela y Nicolao, 2011). Para calcular el pronóstico se empleó la me- todología propuesta por Andriolo, Battini, Grubbström, Persona y Sgarbossa (2014).

La Tabla 1 muestra los datos de ventas del producto durante 14 meses, todos los de 2016 y dos de 2017. Con base en esta serie de tiempo histórica, se estima el nivel de ventas real para usar estos datos como valores futuros.

Tabla 1. Serie de tiempo de las ventas del producto.

\begin{tabular}{|l|l|l|}
\hline \multicolumn{1}{|c|}{ Año } & \multicolumn{1}{|c|}{ Mes } & Ventas en unidades \\
\hline \multirow{4}{*}{} & Enero & 1642570 \\
\cline { 2 - 3 } & Febrero & 2009680 \\
\cline { 2 - 3 } & Marzo & 2173570 \\
\cline { 2 - 3 } & Abril & 2244400 \\
\cline { 2 - 3 } & Mayo & 2409600 \\
\cline { 2 - 3 } 2016 & Junio & 2396980 \\
\cline { 2 - 3 } & Julio & 2206430 \\
\cline { 2 - 3 } & Agosto & 2625060 \\
\cline { 2 - 3 } & Septiembre & 2396790 \\
\cline { 2 - 3 } & Octubre & 2541270 \\
\cline { 2 - 3 } & Noviembre & 2302579 \\
\cline { 2 - 3 } & Diciembre & 2478660 \\
\hline \multirow{4}{*}{2017} & Enero & 2199470 \\
\cline { 2 - 3 } & Febrero & 1334770 \\
\hline
\end{tabular}

Fuente: Elaboración propia.

Para este caso, en la Tabla 2 se eligió como constante de suavizamiento a $\alpha=0,2$ porque el promedio es bastante uniforme y cuenta con pocas probabilidades de cambiar. Además, no presenta picos fluctuantes que requieran un alfa elevado, lo que significa que el resultado del nuevo pronóstico es muy cercano a los datos de las ventas (Morcela y Nicolao, 2011).

En la Figura 1 se observa que las ventas reales fluctúan entre cada mes con picos y valles moderados. Por otro lado, en los primeros dos meses del año las ventas descendieron pero luego se recuperaron. Se desea hallar un pronóstico para el siguiente periodo con error mínimo con el fin de mantener exactitud en las proyecciones del periodo futuro y que varíe a largo plazo alrededor de este promedio. De acuerdo al resultado del modelo EOQ, la empresa puede planear ventas de 2195313 unidades de producto para el periodo siguiente (Lind, Marchal y Wathen, 2012).

\section{Aplicación del modelo EOQ}

El movimiento de la empresa muestra una tasa de demanda conocida y constante, por lo que se aplica el modelo EOQ básico. Para elaborar el producto se realizan tres procesos al día, durante el cual se incurren en gastos como mano de obra, luz, 
Tabla 2. Pronósticos obtenidos por suavizamiento exponencial y de los errores de pronóstico para las ventas del producto ( $\alpha=0,2$ como constante de suavizamiento).

\begin{tabular}{|c|c|c|c|c|}
\hline \multirow{3}{*}{ Año } & Mes $(\mathbf{t})$ & Ventas en unidades $(\mathbf{Y t})$ & $\begin{array}{c}\text { Pronóstico con suavizamiento } \\
\text { exponencial (Ft) }\end{array}$ & Error de pronóstico (Yt-Ft) \\
\hline \multirow{4}{*}{2016} & 1 & 1642570 & & 367110 \\
\cline { 2 - 5 } & 2 & 2009680 & 1642570 & 457578 \\
\cline { 2 - 5 } & 3 & 2173570 & 1715992 & 436892 \\
\cline { 2 - 5 } & 4 & 2244400 & 1807508 & 514714 \\
\cline { 2 - 5 } & 5 & 2409600 & 1894886 & 399151 \\
\cline { 2 - 5 } & 7 & 2396980 & 1997829 & 128771 \\
\cline { 2 - 5 } & 1 & 2206430 & 2077659 & 521647 \\
\cline { 2 - 5 } & 11 & 2625060 & 2103413 & 189047 \\
\cline { 2 - 5 } & 12 & 2396790 & 2207743 & -2117 \\
\hline \multirow{4}{*}{2017} & 13 & 2541270 & 2245552 & 174388 \\
\cline { 2 - 5 } & 14 & 2302579 & 2304696 & -139680 \\
\hline
\end{tabular}

Fuente: Elaboración propia. Pronóstico calculado por suavizamiento exponencial.

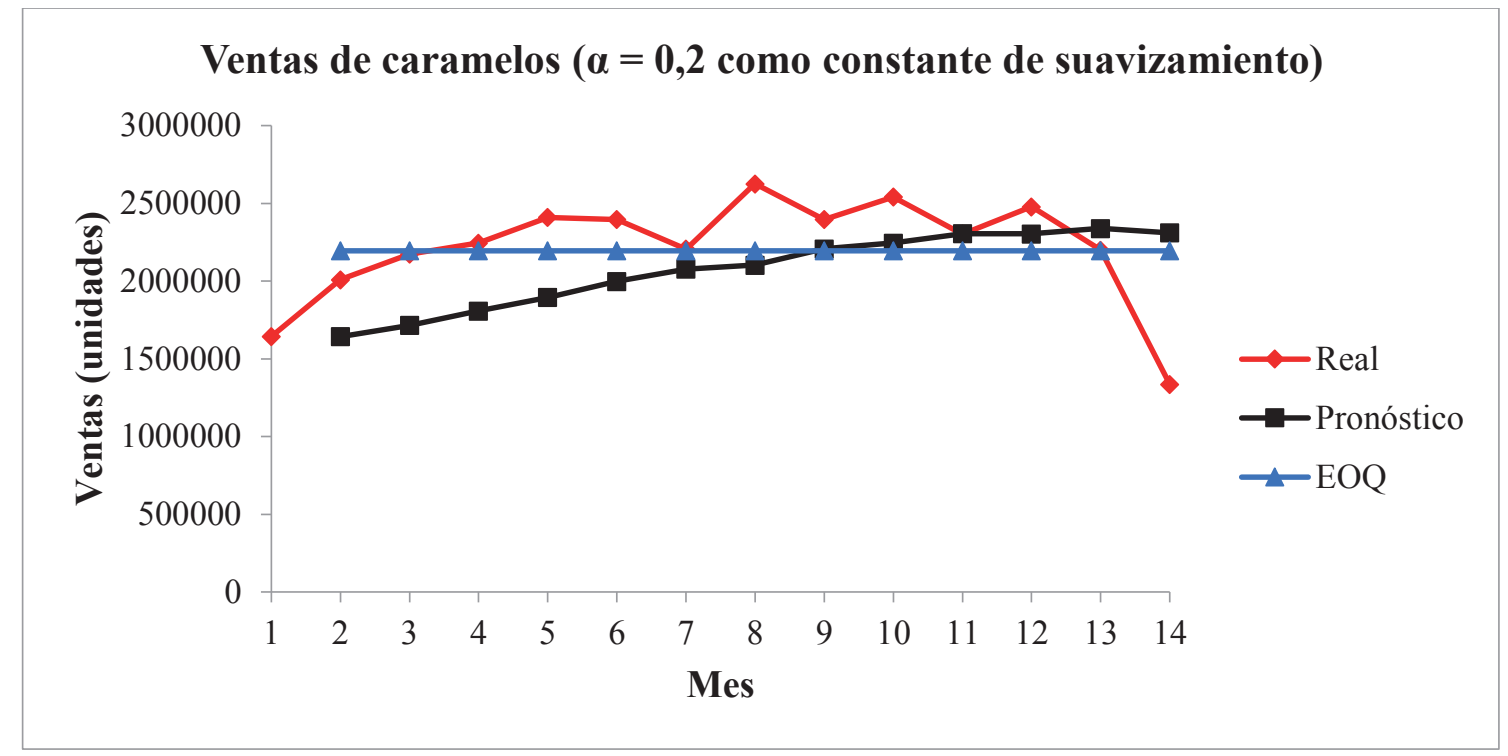

Figura 1. Gráfica de las series de tiempo, real y pronosticada de las ventas de caramelo $(\alpha=0,2$ como constante de suavizamiento).

Fuente: Elaboración propia. Gráfica del pronóstico calculado.

agua, carbón, bolsas, etiquetas, empaques, entre otros, que son variables para calcular la cantidad económica de pedido (EOQ), con el propósito de fabricar un lote óptimo y determinar cuándo y cuántas unidades producir (Taha, 2012). Para realizar los tres procesos al día, se incurre en un costo de preparación de 987462 COP (pesos colombianos), que incluyen los gastos necesarios para ordenar o producir las unidades demandadas. De esta forma, se fabrican 73718 unidades en promedio al día. El costo unitario de producción por unidad es de 29,9 COP, independientemente del tamaño del lote, el cual no incluye el costo de preparación. El tiempo que los productos permanecen almacenados en la 
fábrica es de 1 o máximo 2 días, mientras que el costo de mantener una unidad es de 6,80 COP por día (ver Tabla 3).

Tabla 3. Costos y variables del producto.

\begin{tabular}{|l|l|}
\hline \multicolumn{2}{|c|}{ Variables producto } \\
\hline $\mathrm{K}=$ & $987462 \mathrm{COP}$ \\
\hline $\mathrm{H}=$ & $6,80 \mathrm{COP}$ \\
\hline $\mathrm{D}=$ & $73719,64 \mathrm{COP}$ \\
\hline $\mathrm{C}=$ & $29,9 \mathrm{COP}$ \\
\hline
\end{tabular}

Fuente: Elaboración propia. Variables para calcular el EOQ.

La cantidad económica de pedido (EOQ) óptimo en: $Q=\sqrt{\frac{(2)(73718,64)(987462)}{6,80}}=146354,21$

Tiempo de ciclo:

$$
t=\sqrt{\frac{(2)(987462)}{(73718,64)(6,80)}}=1,99
$$

Punto de reorden:

$$
=(73718,64 / 1)(1)=73718,64
$$

En conclusión, para producir el producto se debe realizar una preparación de producción cada 1,99 días y elaborar 146354,21 unidades cada vez. Así, la solución óptima, redondeando a la unidad más próxima, es producir 146354 unidades cada 2 días o, en consecuencia, cuando el stock baje a 73719 unidades.

\section{El software para el sistema de gestión de inventarios}

Se diseñó una base de datos para llevar los registros de los movimientos del inventario con el fin de tratar grandes volúmenes de información de forma organizada. Por ello, para su diseño se siguió la metodología de Valderrey (2013).

A partir de ello se han definido las cuatro relaciones:

- La relación entre las entidades proveedores y productos tiene el tipo de correspondencia [1:N]; es decir, un proveedor vende uno o más productos $(1, \mathrm{~N})$ y un producto es vendido por uno o varios proveedores $(1, N)$.

- La relación entre las entidades productos y entradas tiene el tipo de correspondencia [1:N]; es decir, un producto tiene cero o más entradas $(0, N)$ y una entrada tiene uno y solo un producto $(1,1)$.

\section{Diagrama E-R}

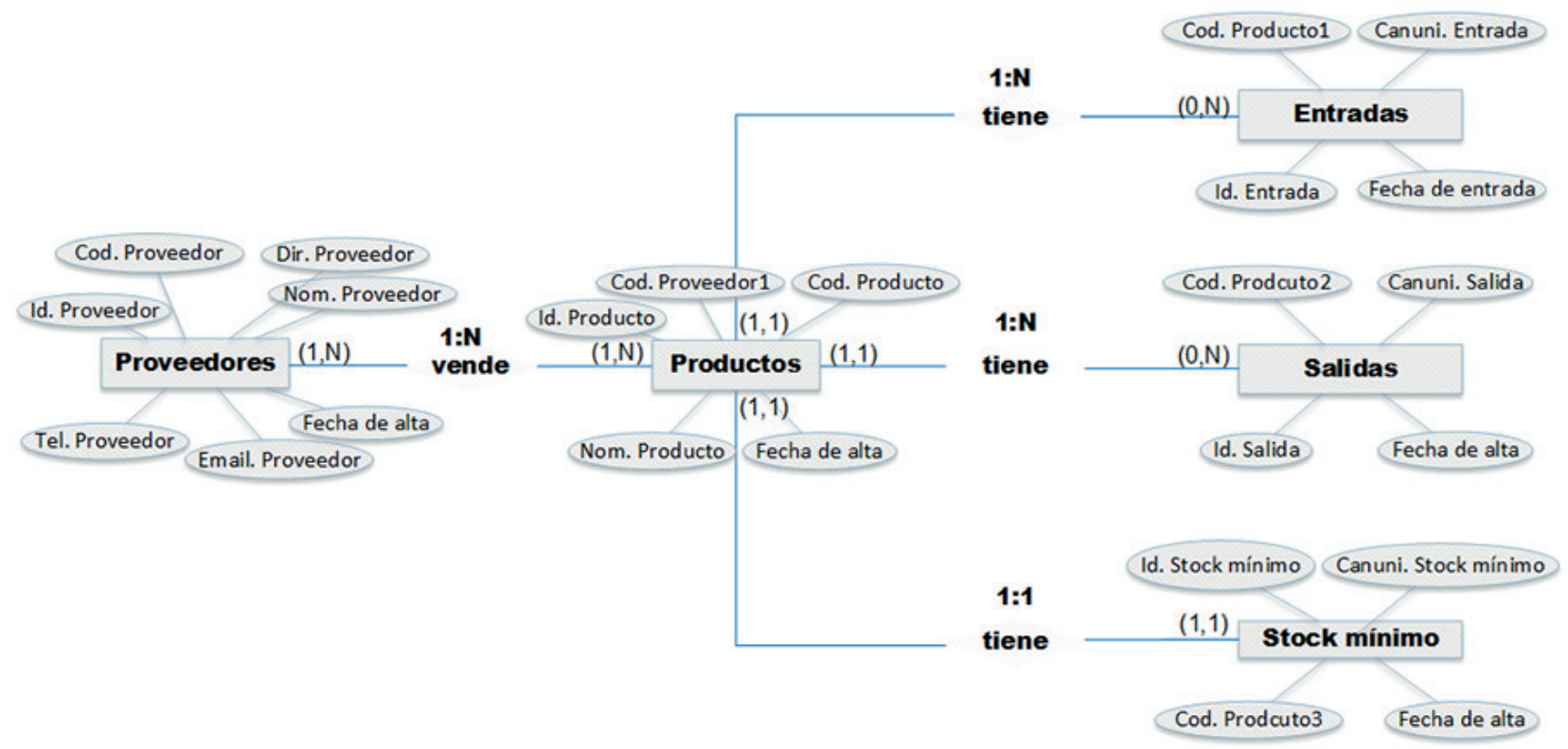

Figura 2. Diagrama entidad-relación de proveedor-producto, producto-entrada, producto-salida y producto-stock mínimo.

Fuente: Elaboración propia. Gráfico de relación proveedor-producto. 
- La relación entre las entidades productos y salidas tiene el tipo de correspondencia [1:N]; es decir, un producto tiene cero o más salidas $(0, N)$ y una salida tiene uno y solo un producto $(1,1)$.

- La relación entre las entidades productos y stock mínimo tiene el tipo de correspondencia [1:1]; es decir, un producto tiene uno y solo un stock mínimo $(1,1)$ y un stock mínimo tiene uno y solo un producto $(1,1)$.

\section{Software: interfaz gráfica del sistema de gestión de inventarios}

Es la parte del sistema que interactúa directamente con el usuario, quien prefiere usar un sistema fácil y sencillo. Por ese motivo, se han utilizado estrategias que favorezcan las relaciones de entradas y salidas de productos (ver Figura 3) y también se ha proyectado una comunicación usuario-sistema más asequible. Asimismo, se ha hecho uso de la ingeniería de software para su diseño y construcción basándose en las facilidades del usuario (Díaz, Harari y Amadeo, 2013).

La Figura 4 presenta un ejemplo de interfaz gráfica de software, cuyo sistema cuenta con varios módu- los que facilitan el estilo de iteración con el usuario, entre los cuales se encuentran el módulo de ingreso, el de inicio, el de proveedores, el de productos, el de stock mínimo, el de generar código QR y el de $E O Q$. De esta forma, cada uno de ellos tiene diferentes funciones que permiten administrar la base de datos, donde destaca el uso del código QR en el módulo de ingreso y en el de stock mínimo, los cuales son suministrados y descargados mediante la lectura de los códigos instalados con anterioridad en los contenedores de materia prima y en las estibas de embalaje final de cada producto.

\section{DISCUSIÓN}

El inventario es parte fundamental de las operaciones de las empresas. Además, es el mecanismo donde la empresa se apoya para realizar sus procesos productivos y poder cumplir con la demanda del mercado. También representa un activo de la empresa; pues, de acuerdo con Osorio (2008), existen organizaciones que no administran sus inventarios de forma correcta, lo cual origina un cálculo erróneo de sus pedidos de reabastecimiento $\mathrm{y}$, en consecuencia, carecen de tiempo suficiente para evitar faltantes.

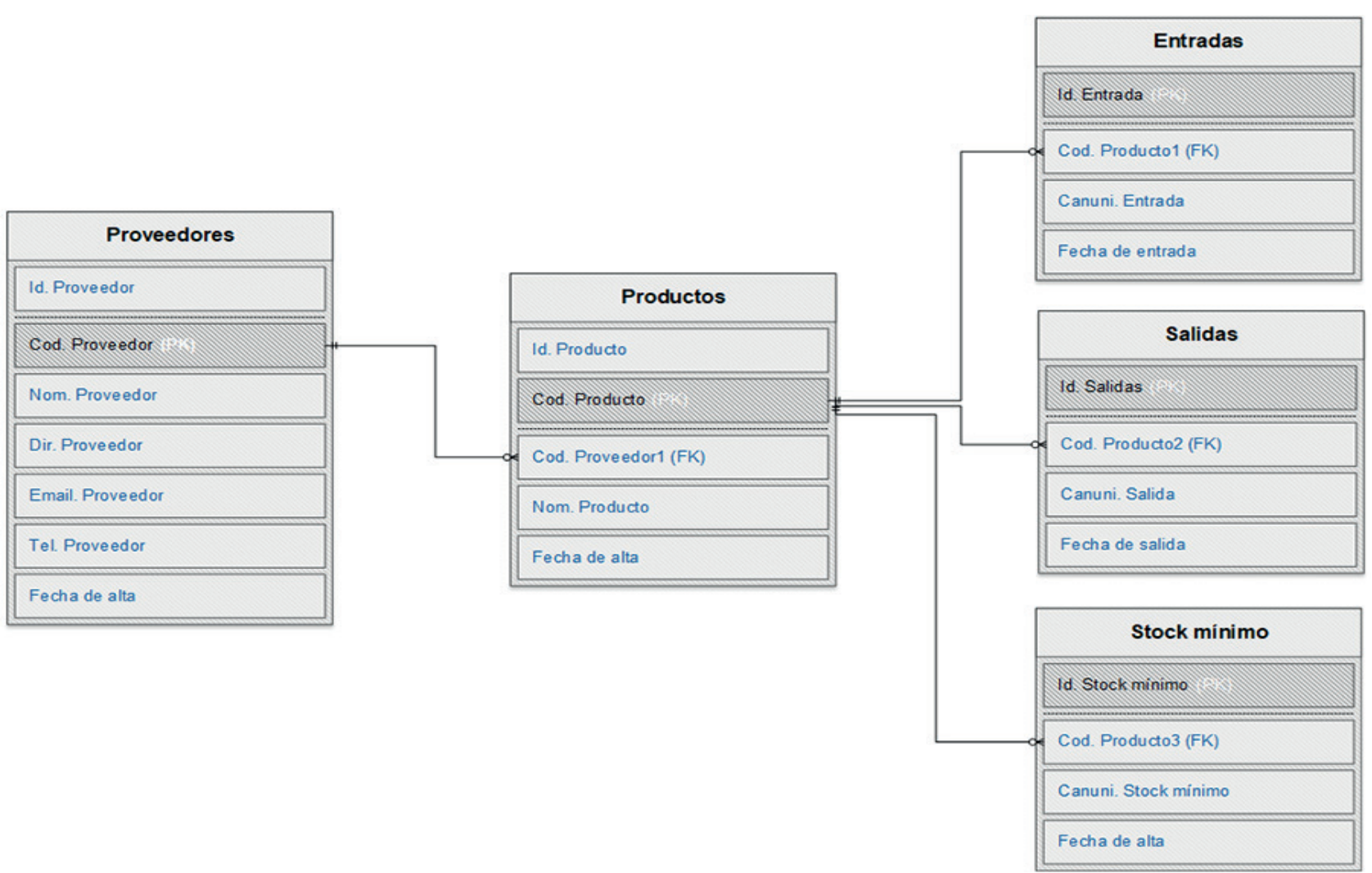

Figura 3. Modelo entidad-relación de proveedor-producto, producto-entrada, producto-salida y producto-stock mínimo. 


\section{ADUIUISTBHCIÓUDEL SISTEMA DE BASES DEDATOS}

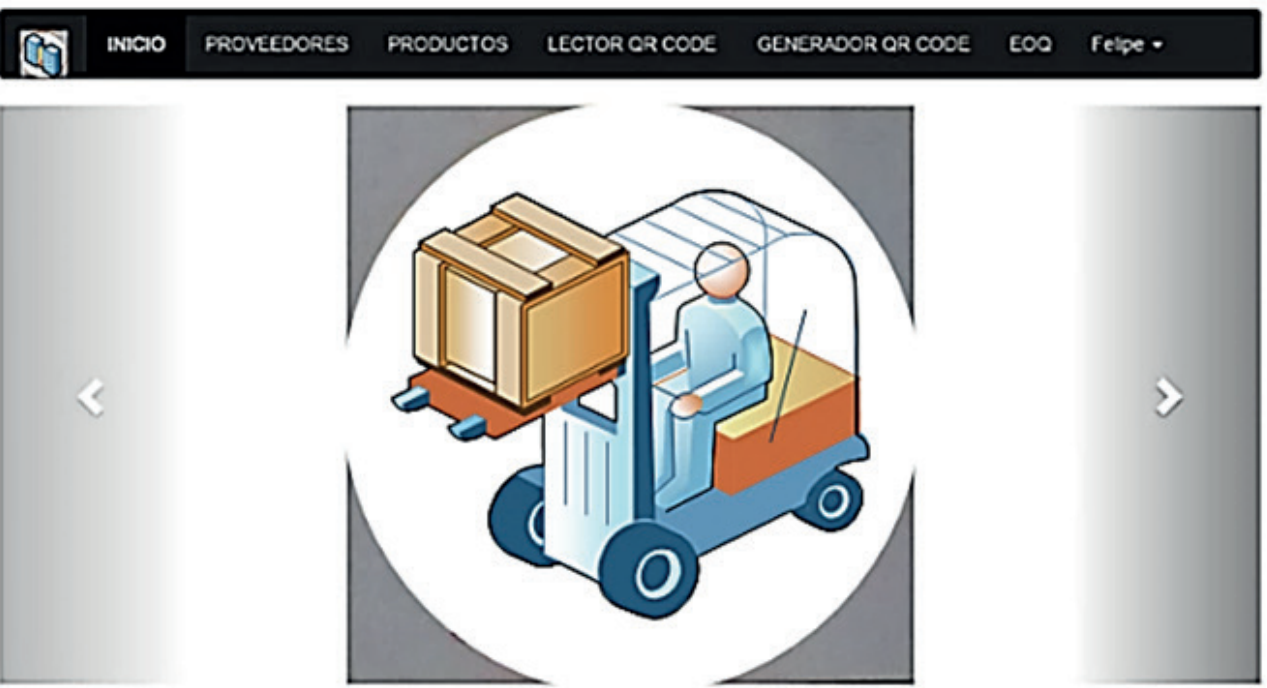

Figura 4. Interfaz gráfica del inicio al sistema.

Fuente: Elaboración propia. Pantalla principal del software.

Entonces, se evidencia que el inventario es el responsable del control de productos en toda organización y lleva registros actualizados de la existencia de un bien o recurso. Asimismo, debe asegurar que su cantidad siempre sea óptima y, de no ser así, tiene que implementar un sistema de inventarios para determinar los niveles ideales. Es relevante saber el momento de restablecer los productos y conocer qué tan grandes deben ser los pedidos; por ello, Chase, Jacobs y Aquilano (2009) mencionan que es de gran utilidad el uso de ayudas tecnológicas que eviten las actividades repetitivas, como el conteo manual del inventario, y permitan la consulta en tiempo real.

Por otro lado, Gutiérrez y Rodríguez (2008) plantean que los sistemas de la información como las TIC deben permitir desarrollar un software a medida de cada organización en particular. En esta investigación, se desarrolla el sistema de control de inventarios vinculado al modelo $E O Q$ con la tecnología (bases de datos, software y código QR) con la finalidad de optimizar el sistema de gestión del inventario.

\section{RECOMENDACIONES}

Se recomienda, a la organización interesada en implementar un sistema de control de inventarios apoyado en códigos QR, realizar una caracterización previa de todas las actividades que la organización lleva a cabo, con el objetivo de controlar sus inventarios, determinar la metodología de gestión más adecuada para la organización en particular y llevar esos requerimientos al software o a las TIC a utilizar.

\section{CONCLUSIONES}

- El sistema de gestión de inventarios es una herramienta muy útil al momento de tomar decisiones que contemplan todas las actividades de aprovisionamiento y distribución de productos.

- El uso del modelo EOQ o de alguna de sus variantes garantiza a la organización una minimización de costos de mantenimiento de inventario, pérdidas por faltantes y escasez de tiempo por actividades de conteo tradicional de productos. Además, se garantiza un punto de reorden que mantendrá bajo control las variables mencionadas y responderá de mejor forma a la demanda del mercado.

- El software, desarrollado para controlar el flujo del inventario con apoyo del código QR, hace más rápido el proceso de administración del inventario y permite almacenar la información de forma segura en una base de datos. Asimismo, permite una posterior consulta, análisis y toma de decisiones en tiempo real. 
- Este mecanismo ha sido diseñado para una organización en particular, en la cual las TIC se acoplaron a los requerimientos de la pyme, como es aconsejable que suceda. Además, funciona como una solución efectiva que no requiere gran capacitación para su uso y responde a las necesidades de la tecnología actual a un bajo costo.

- Finalmente, la implementación del sistema de gestión de inventarios es una respuesta tecnológica a los desafíos de la transformación digital en las organizaciones pequeñas, como las pymes, de manera estratégica y operativa teniendo en cuenta que es una necesidad presente de las entidades del futuro.

\section{REFERENCIAS BIBLIOGRÁFICAS}

[1] Andriolo, A., Battini, D., Grubbström, R., Persona, A. y Sgarbossa, F. (2014). A century of evolution from Harris's basic lot size model: Survey and research agenda. International Journal of Production Economics, 155, 16-38.

[2] Argüello, G. (2015). Uso de las TIC en las PYME del sector industrial del Área Metropolitana de Bucaramanga. I+D Revista de Investigaciones, 6(2), 125-136.

[3] Asociación Nacional de Instituciones Financieras (2018). Gran Encuesta a las Microempresas. Informe de resultados 2018. Recuperado de: http://www.anif.co/sites/ default/files/publicaciones/gem18_.pdf

[4] Chase, R., Jacobs, R. y Aquilano, N. (2009). Administración de operaciones. Producción y cadena de suministros. México D. F., México: McGraw-Hill.

[5] Converse, T., Park, J. y Morgan, C. (2004). PHP5 and MySQL Bible. Indianapolis, Estados Unidos: Wiley Publishing Inc.

[6] Correa, A. y Gómez, R. (2009). Tecnologías de la información en la cadena de suministro. DINA, 76(157), 37-48.

[7] Correa, A., Álvarez, C. y Gómez, R. (2010). Sistemas de identificación por radiofrecuencia, código de barras y su relación con la gestión de la cadena de suministro. Estudios Gerenciales, 26(116), 115-141.

[8] Díaz, F., Harari, I. y Amadeo, A. (2013). Guía de recomendaciones para diseño de software centrado en el usuario. La Plata, Argentina: Editorial de la Universidad de La Plata.
[9] Duong, L., Wood, L. y Wang, W. (2015). A Multicriteria Inventory Management System for Perishable \& Substitutable Products. Procedia Manufacturing, 2, 66-76.

[10] Gauchat, J. (2012). El gran libro de HTML5, CSS3 y Javascript. Barcelona, España: Marcombo Ediciones Técnicas.

[11] Gutiérrez, V. y Rodríguez, L. (2008). Diagnóstico regional de gestión de inventarios en la industria de producción y distribución de bienes. Revista Facultad de Ingenieria Uniersidad de. Antioquía, (45), 157-171.

[12] Hernández, R., Fernández, C. y Baptista, P. (2014). Metodología de la investigación. México D. F., México: McGraw-Hill.

[13] Hillier, F. y Lieberman, G. (2010). Introducción a la investigación de operaciones. México D. F., México: McGraw-Hill.

[14] Lind, D., Marchal, W. y Wathen, S. (2012). Estadística aplicada a los negocios y la economía. México D. F., México: McGraw-Hill.

[15] Morcela, O. y Nicolao, J. (2011). Programación maestra de la producción con gestión de inventarios de productos terminados para PyMEs. Caso de aplicación: PyME local, sector alimentos congelados. (Tesis de pregrado). Universidad Nacional de Mar de Plata, La Plata.

[16] Osorio, C. (2008). Modelos para el control de inventarios en las pymes. Panorama, 2(6), 4-10.

[17] Pérez, R., Mosquera, S. y Bravo, J. (2012). Aplicación de modelos de pronósticos en productos de consumo masivo. Biotecnología en el Sector Agropecuario y Agroindustrial, 10(2), 117-125.

[18] Piña, J. (2012). Determinación de la cantidad económica de pedido en una empresa cauchera venezolana aplicando la técnica LIMIT. Ingeniería Industrial. Actualidad y Nuevas Tendencias, 3(9), 61-72.

[19] Pressman, R. (2010). Ingeniería del software. Un enfoque práctico. México D. F., México: McGraw-Hill.

[20] Render, B. y Heizer, J. (2004). Principios de administración de operaciones. México D. F., México: Pearson Educación.

[21] Quigley, E., y Gargenta, M. (2007). PHP and MySQL by example. Massachusetts, Estados Unidos: Pearson Education Inc. 
[22] Quintero, J. (2018). Las pymes en Colombia y las barreras para su desarrollo y perdurabilidad. (Tesis de pregrado). Universidad Militar Nueva Granada, Bogotá.

[23] Regalado, R. (2007). Las MIPYMES en Latinoamérica. S. I.: Organización Latinoamericana de Administración.

[24] Sandoval, A. (2016). Uso de códigos QR en unidades didácticas. Revista de Posgrado y Sociedad. Sistema de Estudios de Posgrado, 14(1), 39-47.

[25] Servera, F., Gil, I. y Fuentes, M. (2009). La influencia de la calidad de servicio logístico en la lealtad: un análisis del papel moderador de las TIC. Investigaciones Europeas de Dirección y Economía de La Empresa, 15(3), 37-58.

[26] Taha, H. (2012). Investigación de Operaciones. México D. F., México: Pearson.

[27] Valderrey, P. (2013). Administración de sistemas gestores de bases de datos. Madrid, España: Ecoe Ediciones.

[28] Vila, J. (2011). Los códigos QR aplicados a la educación. Comunicación y pedagogía: Nuevas tecnologías y recursos didácticos, (253), 27-29. 


\section{System design for inventory management of SMEs in the food industry}

\begin{abstract}
This paper provides a proposal designed to improve the inventory management system of SMEs, especially those in the candy production and sales sector. The objective of the study is to increase productivity in terms of management and control of inventories; therefore, the methodology consists of carrying out a bibliographic review about the context and challenges presented by SMEs related to the application of ICTs. Then, the diagnosis of the company under study will make it possible to calculate the forecast based on the most accurate procedure identified in the previous phase. In conclusion, the aim is to apply an inventory control system using software designed specifically for this company, which will use QR codes for data supply and updating in real time.
\end{abstract}

Keywords: Economic order quantity; QR code; inventory control; inventory management; inventory system.

\section{INTRODUCTION}

First and foremost, should be noted that inventory is a fundamental part of an organization's operations, since it creates a record of commercial and productive activities. Therefore, it must be performed in such a way that prevents product oversupply, which would cost the company a large sum of money. At the same time, the manager must calculate orders through the inventory so that there are no shortages. For this reason, Hillier and Lieberman (2010) note that "[...]inventories pervade the business world. Maintaining inventories is necessary for any company dealing with physical products, including manufacturers, wholesalers, and retailers" (p. 772).

In Colombia, approximate figures indicate that SMEs:

[...] constituyen más del $90 \%$ de las entidades económicas, el $75 \%$ de los nuevos puestos de empleo y el $53 \%$ de la producción bruta de los sectores industrial, comercial y de servicios se convierten en parte de la esencia de la economía nacional, no sólo por el volumen de este tipo de empresas, sino también por la relevancia que tienen en la contribución a los índices de empleo del país. [constitute more than $90 \%$ of economic entities, $75 \%$ of new jobs and $53 \%$ of gross industrial output, commercial and service sectors, which become an essential part of the national economy, not only for the size of these businesses, but also for the relevance they have on employment rates in the country.] (Quintero, 2018, p. 12)

In this way, data such as those provided by the Gran Encuesta a las Microempresas [Great Survey of Microenterprises], prepared by the Asociación Nacional de Instituciones Financieras [Colombian National Association of Financial Institutions] (2018), reveal

1 Master's in Design and Process Management and research professor at the Universidad Pedagógica y Tecnológica de Colombia. Boyacá, Colombia.

E-mail: diego.carreno@uptc.edu.co

2 Specialist in Production and Operations Engineering and research professor at the Universidad Pedagógica y Tecnológica de Colombia. Boyacá, Colombia.

E-mail: luisfelipe.amaya@uptc.edu.co

3 Master's degree in Industrial Engineering and research professor at the Universidad Pedagógica y Tecnológica de Colombia. Boyacá, Colombia. E-mail: erika.ruiz03@uptc.edu.co

4 Industrial manager and researcher at the Universidad Pedagógica y Tecnológica de Colombia Boyacá, Colombia.

E-mail: felipe.tiboche@uptc.edu.co 
the most common problems faced by these small and medium enterprises. Some of them have also been demonstrated in the study by Regalado (2007), which notes "acceso restringido a las fuentes de financiamiento, bajos niveles de capacitación de sus recursos humanos, limitados niveles de innovación y desarrollo tecnológico, baja penetración en mercados internacionales, bajos niveles de productividad, baja capacidad de asociación y administrativa" [restricted access to sources of financing, low levels of human resources training, limited levels of innovation and technological development, low foreign market entry, low levels of productivity, low capacity for association and administration] (p. 73).

Likewise, previous investigations have shown problems that have persisted over time, as shown in the study by Gutiérrez \& Rodríguez (2008), which concludes that SMEs in Colombia do not have a lost sales control system that make it possible to measure the level of service rendered in order to determine the optimal inventory level of each finished product. Also analyzed was the fact that most companies make decisions based on experience and management models supported by qualitative techniques and, to a lesser extent, based on statistical methods of forecasting demand. In addition, it was found that $10 \%$ of the companies that implemented some type of technology to help control the organization inventory had to adapt their processes to the requirements of the software, when the opposite should occur.

All the drawbacks mentioned in the previous paragraph can be solved - notes Argüello (2015) in his diagnosis of SMEs inventory management in the industrial sector - through efficient inventory management. According to the proposal, it is established that ICTs play a key role in inventory management, since there are different IT tools in the market to support supply chain management. Some of them are: VMI (Vendor Managed Inventory), CPFR (Collaborative Planning Forecasting and Replenishment), RFID (Radio Frequency Identification) and bar codes, which are used for identification, registration of operations and traceability, and WMS (Warehouse Management System) and LMS (Labor Management System), which support the management of information flows when performed correctly (Correa \& Gómez, 2009).

Despite the support that IT tools provide, Colombian SMEs claim that they do not implement any ICT due to lack of knowledge or investment capacity; while those that decide to apply them do not have personnel with ICT knowledge or the skills to implement them (Correa, Álvarez \& Gómez, 2010; Argüello, 2015).

On the other hand, according to Servera, Gil and Fuentes (2009), ICTs play a key role in the quality of the inventory management process of companies, since in their research they show the relevance of conducting a study on these, applied to the function of logistics and raw material management. Thus, the rapid incorporation of advances in ICTs into logistics is a differential factor that makes it possible to improve the efficiency of all processes because it generates value for the client.

From the point of view of Correa, Álvarez and Gómez (2010), the maximum degree of inventory control is achieved in companies with the implementation of automated control and identification systems for products, such as "las tecnologías de código de barras y la radiofrecuencia (RFID) [que] son sistemas de identificación de productos y captura de datos que contribuyen a que los flujos de información en la gestión de la cadena de suministro se realicen de manera eficiente y ágil" [barcode technologies and Radio Frequency (RFID) [which] are product identification and data capture systems that contribute in making the information in supply chain management flow efficiently and swiftly] (pp. 117-118). However, these systems present storage problems and erroneous readings due to technology readiness level of current technology; therefore, Duong, Wood and Wang (2015) propose two options: use both systems simultaneously or seek new technological alternatives that are faster, free, adjustable and of lower technological cost. From this, a new storage option for inventory management emerges: QR (Quick Response) codes. According to Sandoval (2016), these were created by Japanese company Denso Wave for logistic and inventory control purposes, since "los códigos $Q R$ son un mobile tag, es decir, un conjunto de imágenes bidimensionales que se descodifican con un teléfono móvil y que permiten dar información más directa al usuario" [QR codes are a mobile tag, that is, a set of two-dimensional images that are decoded with a mobile phone and make it possible to provide more direct information to the user] (Vila, 2011, p. 1).

Although all these ICTs must be supported by an inventory control methodology, authors like Osorio (2008) recommend several inventory management systems for SMEs, which he organizes in order of difficulty and implementation: "control manual, sistema Two-Bin, revisión visual, puntos de reorden $E O Q$, puntos de reorden con lotes dependientes de inventario, revisión periódica con lotes dependientes del nivel de inventario y revisión periódica 
con puntos de reorden y lotes dependientes del nivel de inventario" [Manual control, Two-bin system, visual review, EOQ reorder point, reorder points with inventory dependent sets, periodic review with sets dependent on the inventory level and periodic review with reorder points and sets dependent on the inventory level] (p.6).

To design the inventory management system, in the case of this study, software capable of calculating the economic order quantity, generating QR codes and managing the information in a database was developed. With this application, missing data will be avoided, storage and personnel costs will be reduced, time will be saved, inventory levels will be reduced and the optimal order quantity will be planned. This will be achieved through an affordable standard reader that updates stock information in real time (Hillier \& Lieberman, 2010).

\section{METHODOLOGY}

First, the interview questionnaire for the manager, the accountant and the production supervisor of the company is prepared in order to collect the most relevant variables of the inventory value chain. Subsequently, the data obtained in the interviews are tabulated, completed in the same way as Hernández, Fernández and Baptista (2014). Lastly, it is verified that the inventory level of the company is not too high, since economic losses due to fluctuations in demand should be avoided. Thus, the company should ideally maintain the inventory stock at the optimal level (Render \& Heizer, 2004).

The inventory management system should be designed according to the needs of the company, since these have been identified in the initial diagnosis and are based on the theories of authors who have written about inventory management. In this way, the inventory consists of the units of a good such as the raw material, the product in process and the finished product of the organization, which are available for use in the warehouse or in the production plant. In that sense, Chase, Jacobs and Aquilano (2009) define the inventory as "las existencias de una pieza o recurso utilizado en una organización" [the stock of a piece or resource used in an organization]. They also point out that "un sistema de inventario es el conjunto de políticas y controles que vigilan los niveles del inventario y determinan aquellos a mantener, el momento en que es necesario reabastecerlo y [saber] qué tan grandes deben ser los pedidos" [an inventory system is the set of policies and controls that monitor inventory levels and determine which ones to maintain, when it is necessary to restock and [know] how large the orders must be] (p. 547).

The demand forecasting is calculated with the exponential smoothing model, which is the most common forecasting technique and an integral part of almost all computer forecasting programs; for this reason, they are used very frequently when ordering inventory in retail companies, wholesalers and perishable products retailers. In this way, the research by Pérez, Mosquera and Bravo (2012) uses this practice to compare the utility between the different forecasting methods in mass consumption products of the family shopping basket. The conclusion reached is that the exponential smoothing method responds well when the sales trend is growing, as presented in this case study.

The inventory control methodology selected was the model of economic order quantity (EOQ), selected from the study by Osorio (2008), which recommends that the inventory management models be adapted to Colombian SMEs. The EOQ model is part of the fourth complexity after the manual control models, Two-Bin system and visual review, used before in the analyzed company. In addition, due to the good results obtained in his research, Piña (2012) recommends this control system in companies with perishable raw materials. The EOQ model flourished from about 1913 and was one of the pioneering models in inventory control (Andriolo, Battini, Grubbström, Persona \& Sgarbossa, 2014).

The equation (1), proposed by Hillier and Lieberman (2010), represents the EOQ formula, used to calculate the optimal quantity of products to be ordered or produced by lot.

$$
Q=\sqrt{\frac{2 d K}{h}}
$$

Also, equation (2) corresponds to the cycle time.

$$
t=\sqrt{\frac{2 K}{d h}}
$$

Reorder point $=($ demand rate $) \times($ Delivery time $)$

Where:

$\mathrm{K}=$ preparation cost to order a lot,

$\mathrm{d}=$ demand rate, and

$h=$ cost to maintain the inventory per unit of time.

The design and programming of software are necessary processes for the creation of information 
systems within organizations, since they make it possible to manage information very effectively and handle data quickly, reliably, safely, in a traceable manner and in real time. According to Pressman (2010), "el software se ha incrustado profundamente en casi todos los aspectos de nuestras vidas" [software has become deeply embedded in almost every aspect of our lives] (p.10); so before developing software, it is necessary to fully understand the problem that needs to be solved.

For Quigley and Gargenta (2007), the software designed encompasses the specifications and structure of the inventory management system required by the organization, which is based on the QR codes reading for feeding and updating data (Duong, Wood \& Wang, 2015). Being a technology that can store more information than bar codes, it allows a greater speed with today's computer systems and meets the specifications required by a small company.

Taking Pressman (2010) as a starting point, who states that the software must include a set of written programs, it is certified that the languages used to program the inventory management system are PHP (Hypertext Preprocessor). According to Converse, Park and Morgan (2004), these are free software, guaranteed by a set of licensing schemes that do not have copyleft or copyright restrictions, that is, it is an open source language, as pointed out by Quigley and Gargenta (2007). On the other hand, HTML means HyperText Markup Language, which uses a tag language tags to build web pages (Gauchat, 2012). From another angle, CSS are cascading style sheets, which is a simple mechanism for adding styles, for example, fonts, colors or spacing, to web documents (Quigley \& Gargenta, 2007). On the other hand, JavaScript $\AA$ is an interpreted language, dynamic object-oriented programming, which is used in millions of web pages and server applications throughout the world (Valderrey, 2013). Finally, Quigley and Gargenta (2007) indicate that the database management system called MySQL is "the world's most popular open source database" (p.7), since it is powered by a standard QR reader that is part of innovation and optimization in costs and time. Thus, any operator that has a smartphone or iPhone can update this information, which will avoid a production stoppage due to manual or visual inventory management.

\section{RESULTS}

\section{Product sales forecast}

On the one hand, the smoothing method was very accurate on the forecast for the following period, while the time series chart allowed us to investigate trends in the data that are optimized over a period of time (Morcela \& Nicolao, 2011). To calculate the prognosis, the methodology proposed by Andriolo, Battini, Grubbström, Persona and Sgarbossa (2014) was used.

Table 1 shows the product sales data for 14 months, all of 2016 and two months of 2017. Based on this historical time series, the level of actual sales is estimated in order to use these numbers as future values.

Table 1. Product sales time series.

\begin{tabular}{|c|c|c|}
\hline Year & Month & Sales in units \\
\hline \multirow{4}{*}{2016} & January & 1642570 \\
\cline { 2 - 3 } & February & 2009680 \\
\cline { 2 - 3 } & March & 2173570 \\
\cline { 2 - 3 } & April & 2244400 \\
\cline { 2 - 3 } & May & 2409600 \\
\cline { 2 - 3 } & June & 2396980 \\
\cline { 2 - 3 } & July & 2206430 \\
\cline { 2 - 3 } & August & 2625060 \\
\cline { 2 - 3 } & September & 2396790 \\
\cline { 2 - 3 } & October & 2541270 \\
\cline { 2 - 3 } & November & 2302579 \\
\hline \multirow{4}{*}{2017} & December & 2478660 \\
\cline { 2 - 3 } & January & 2199470 \\
\cline { 2 - 3 } & February & 1334770 \\
\hline
\end{tabular}

Source: Prepared by the authors.

In Table 2, $\alpha=0.2$ was chosen as a smoothing constant because the average is fairly uniform and has little probability of changing. In addition, it does not present fluctuating peaks that require a high alpha, which means that the result of the new forecast is very close to the sales data (Morcela \& Nicolao, 2011).

Figure 1 shows that actual sales fluctuate each month with moderate peaks and valleys. On the other hand, sales fell in the first two months of the year but then recovered. A forecast for the next period with minimum error is sought in order to maintain accuracy in the forecast of the future period, that varies in the long term around this average. According to the EOQ model results, the company can plan sales of 2195313 product units for the following period (Lind, Marchal \& Wathen, 2012).

\section{EOQ model application}

The movement in the company shows a known and constant demand rate, which is why the basic $E O Q$ 
Table 2. Forecasts obtained by exponential smoothing and forecast errors for product sales $(\alpha=0.2$ as smoothing constant).

\begin{tabular}{|c|c|c|c|c|}
\hline Year & Month (t) & Sales in units (Yt) & $\begin{array}{c}\text { Forecast with exponential } \\
\text { smoothing (Ft) }\end{array}$ & Forecast error (Yt-Ft) \\
\hline \multirow{5}{*}{} & 1 & 1642570 & & 367110 \\
\cline { 2 - 5 } & 2 & 2009680 & 1642570 & 457578 \\
\cline { 2 - 5 } & 3 & 2173570 & 1715992 & 436892 \\
\cline { 2 - 5 } & 4 & 2244400 & 1807508 & 514714 \\
\cline { 2 - 5 } & 5 & 2409600 & 1894886 & 399151 \\
\cline { 2 - 5 } & 6 & 2396980 & 1997829 & 128771 \\
\cline { 2 - 5 } & 7 & 2206430 & 2077659 & 521647 \\
\cline { 2 - 5 } & 9 & 2625060 & 2103413 & 295718 \\
\cline { 2 - 5 } & 10 & 2396790 & 2207743 & -2117 \\
\cline { 2 - 5 } & 11 & 2541270 & 2245552 & 174388 \\
\hline \multirow{4}{*}{2017} & 12 & 2302579 & 2304696 & -139680 \\
\cline { 2 - 5 } & 14 & 2478660 & 2304272 & -976444 \\
\hline
\end{tabular}

Source: Prepared by the authors. Forecast calculated by exponential smoothing.

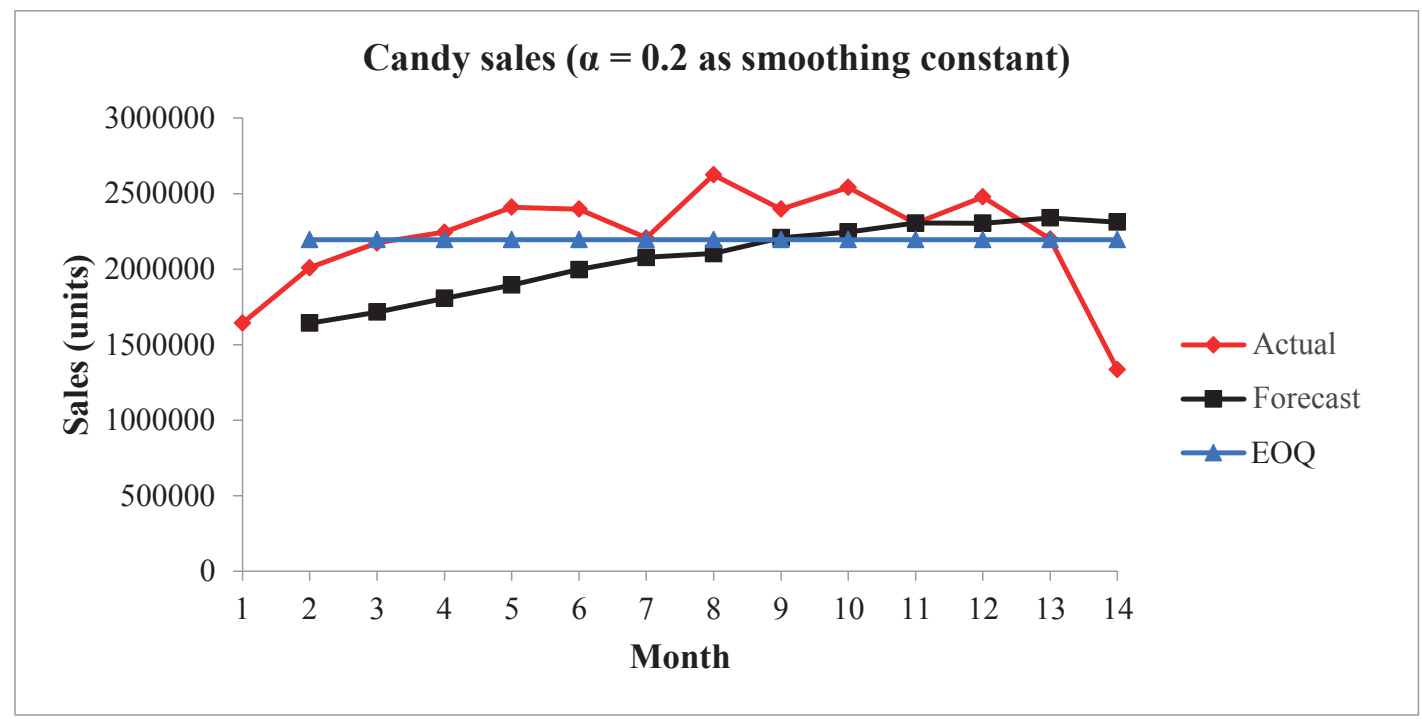

Figure 1. Time series, real and forecasted, candy sales chart ( $\alpha=0.2$ as smoothing constant).

Source: Prepared by the authors. Calculated forecast chart.

model is applied. To manufacture the product, three processes are carried out per day, during which expenses such as labor, light, water, coal, bags, labels, packaging, among others, are incurred, which are variables to calculate the economic quantity of the order (EOQ), with the purpose of producing an optimal lot and determining when and how many units to produce (Taha, 2012). To carry out the three processes per day, a preparation cost of 987
462 COP (Colombian pesos) is incurred, which includes the expenses necessary to order or produce the demanded units. In this way, 73718 units are manufactured on average per day. The unit cost of production is $29.9 \mathrm{COP}$, regardless of the lot size, which does not include the cost of preparation. The products remain stored in the factory for 1 or maximum 2 days, and the maintaining cost of a unit is 6.80 COP per day (see Table 3). 
Table 3. Product costs and variables.

\begin{tabular}{|l|l|}
\hline \multicolumn{2}{|c|}{ Product variables } \\
\hline $\mathrm{K}=$ & $987462 \mathrm{COP}$ \\
\hline $\mathrm{H}=$ & $6.80 \mathrm{COP}$ \\
\hline $\mathrm{D}=$ & $73719.64 \mathrm{COP}$ \\
\hline $\mathrm{C}=$ & $29.9 \mathrm{COP}$ \\
\hline
\end{tabular}

Source: Prepared by the author. Variables to calculate EOQ.

The optimal economic order quantity $(E O Q)$ in:

$$
Q=\sqrt{\frac{(2)(73718.64)(987462)}{6.80}}=146354.21
$$

Cycle time:

$$
t=\sqrt{\frac{(2)(987462)}{(73718.64)(6.80)}}=1.99
$$

Reorder point:

$$
=(73718.64 / 1)(1)=73718.64
$$

In conclusion, to manufacture the product, a production preparation should be carried out every 1.99 days and 146354.21 units should be prepared each time. Thus, the optimal solution, rounding to the nearest unit, is to produce 146354 units every 2 days or, consequently, when the stock drops to 73 719 units.

\section{The software for the inventory management system}

A database was designed to keep records of inventory movements in order to handle large volumes of information in an organized manner. For this reason, its design followed the methodology of Valderrey (2013).

From this, four relationships have been defined:

- The relationship between supplier and products entities has the type of correspondence [1:N]; that is, a supplier sells one or more products $(1, \mathrm{~N})$ and a product is sold by one or more suppliers $(1, N)$.

- The relationship between products and input entities has the correspondence type [1:N]; that is, a product has zero or more inputs $(0, N)$ and one input has one and only one product $(1,1)$.

- The relationship between the products and output entities has the correspondence type [1:N]; that is, a product has zero or more

\section{ER Diagram}

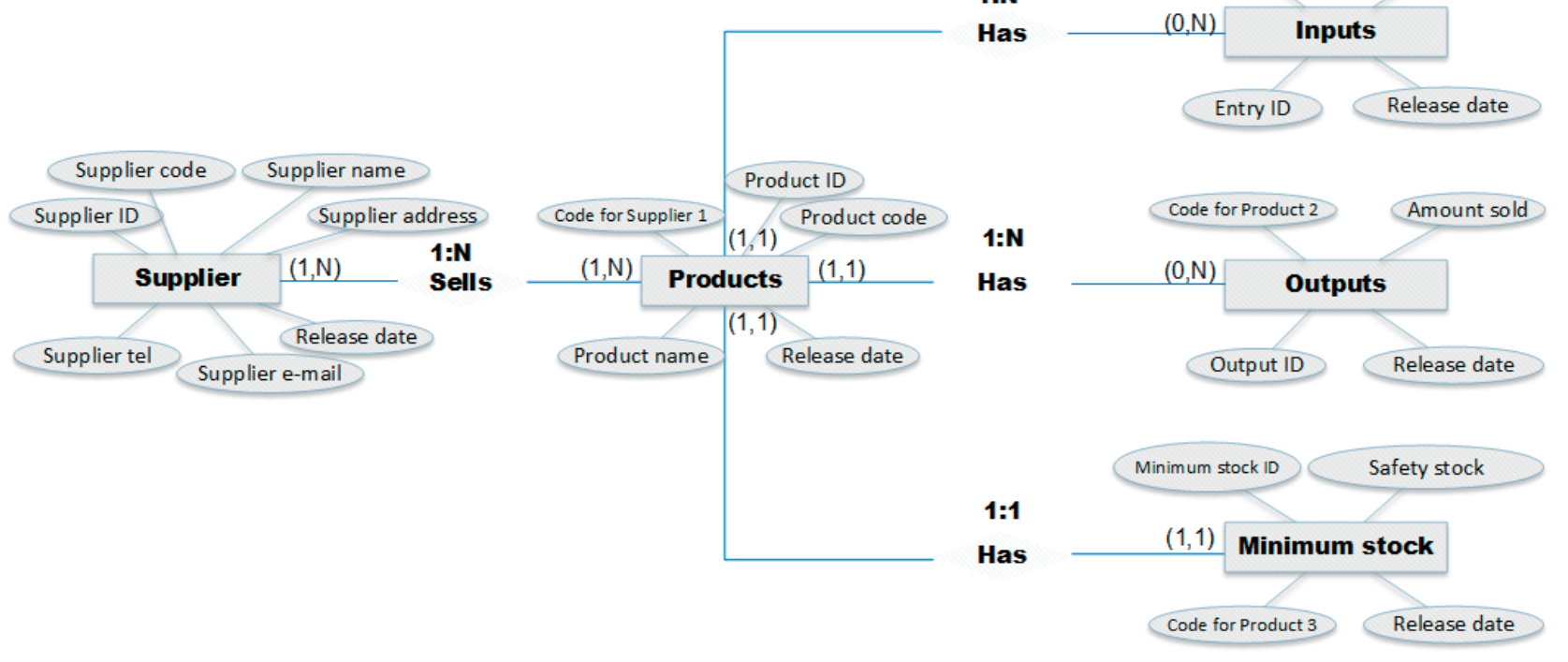

Figure 2. Entity-relationship diagram of supplier-product, product-input, product-output and minimum stock-product.

Source: Prepared by the authors. Supplier-product relationship diagram. 
outputs $(0, N)$ and an output has at most one product $(1,1)$.

- The relationship between the products and minimum stock has the correspondence type [1:1]; that is, a product has one and only a minimum stock $(1,1)$ and a minimum stock has at most one product $(1,1)$.

\section{Software: graphic interface of the inventory management system}

This is the part of the system that interacts directly with the user, who prefers to use an easy and simple system. For this reason, strategies have been used to favor the products inputs and outputs relations (see Figure 3) and a more accessible user-system communication has also been projected. Likewise, software engineering has been used for its design and construction based on the easiness for the user (Díaz, Harari \& Amadeo, 2013).

Figure 4 shows an example of a software graphical interface, whose system has several modules that facilitate the iteration style with the user, among which are the entry, the startup, the vendor, the product, the minimum stock, the QR code and the EOQ modules. In this way, each of them has dif- ferent functions that allow managing the database, where the use of the QR code in the input and the minimum stock modules is highlighted, which are supplied and downloaded by reading the codes previously installed in the raw material containers and in the final dunnage of each product.

\section{DISCUSSION}

The inventory is a fundamental part of the company's operations. In addition, it is the mechanism where the company relies to carry out its production processes and to be able to meet market demand. It also represents a company asset; due to the fact that, according to Osorio (2008), there are organizations that do not manage their inventories correctly, which leads to an erroneous calculation of their replenishment requests and, consequently, lack of sufficient time to avoid shortages.

Therefore, it is demonstrated that the inventory is responsible for product control in every organization and keeps updated records of the existence of a good or resource. Also, it must ensure that the quantity is always optimal and, if not, an inventory system has to be implemented to determine the ide-

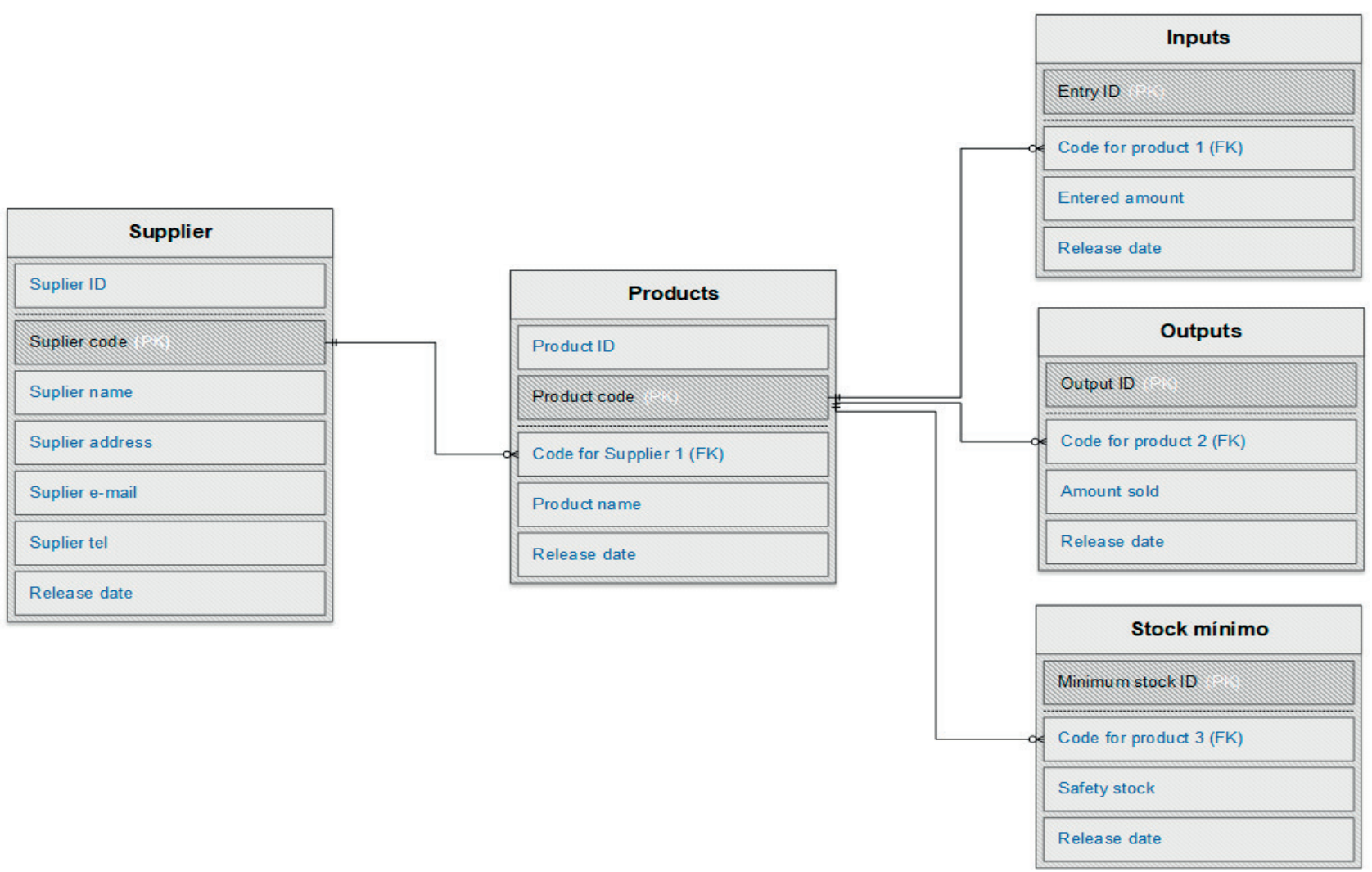

Figure 3. Entity-relationship diagram of supplier-product, product-input, product-output and minimum stock-product. Source: Prepared by the authors. Products inputs and outputs relationship. 


\section{ADHLISTRIIOH OF THE DILIBISE SYSTEII}

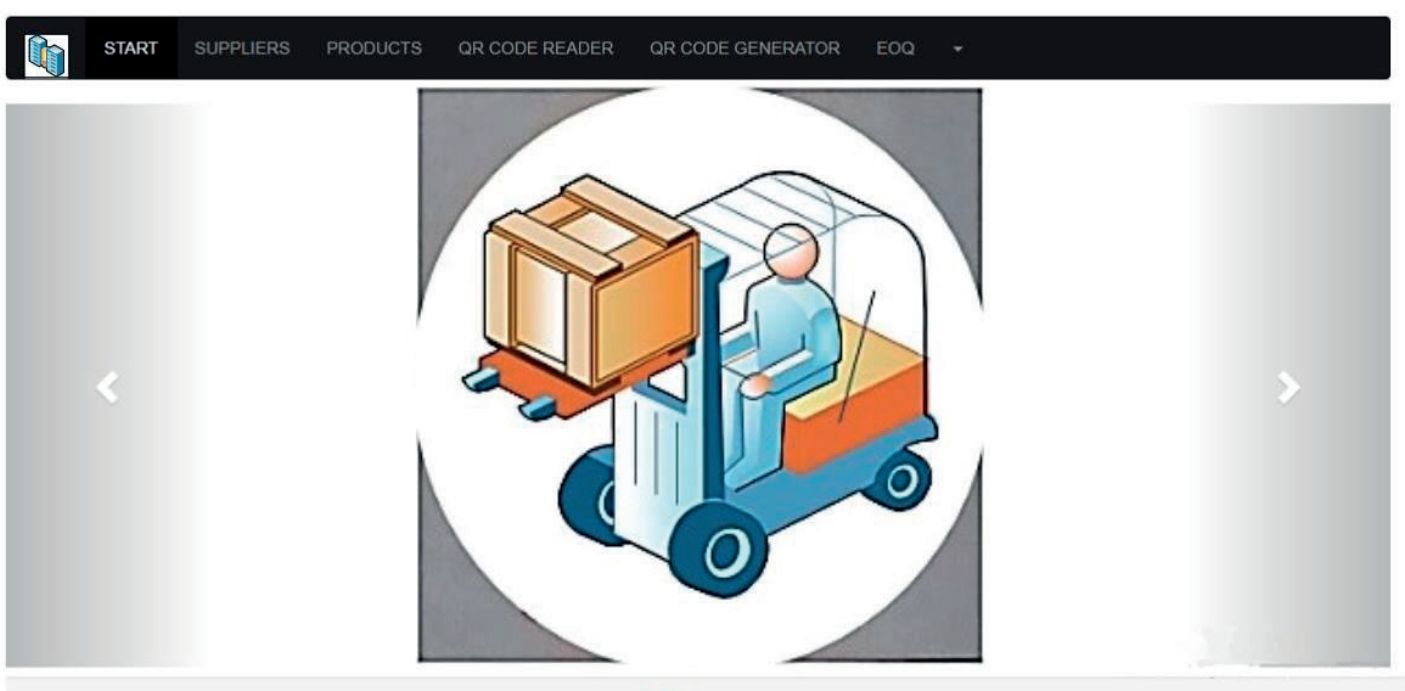

Figure 4. Graphic interface of the system startup.

Source: Prepared by the authors. Software main screen.

al levels. It is relevant to know when to restock the products and to know how large the orders should be; therefore, Chase, Jacobs and Aquilano (2009) mention that it is very useful to use technological aids that avoid repetitive activities, such as manual inventory counting, and make possible the use of real-time consultation.

On the other hand, Gutiérrez and Rodríguez (2008) suggest that information systems such as ICTs should allow for the development of software tailored for each particular organization. In this study, the inventory control system linked to the $E O Q$ model is developed with the technology (databases, software and QR code) that optimizes the inventory management system.

\section{RECOMMENDATIONS}

It is recommended that the organization interested in implementing an inventory control system supported by QR codes carry out a preliminary analysis of all the activities carried out by the organization in order to control their inventories, determine the most appropriate management methodology for the organization in particular and bring those requirements to the software or the ICT to be used.

\section{CONCLUSIONS}

- The inventory management system is a very useful tool when making decisions that include all the activities of product supply and distribution.

- The use of the EOQ model or any of its variants guarantees to the organization a minimization of inventory maintenance costs, losses due to shortages and time lost due to traditional product counting activities. In addition, a reorder point that will keep the aforementioned variables under control and will respond better to market demand is guaranteed.

- The software developed to control the inventory flow with QR code support makes the process of inventory management faster and makes it possible to store information safely in a database. Likewise, it enables a subsequent consultation, analysis and decision making in real time.

- This mechanism has been designed for a particular organization, in which ICTs were adapted to the requirements of the SME, as advisable. In addition, it works as an effective solution that does not require extensive training for its use and responds to the needs of current technology at a low cost.

- Finally, the implementation of the inventory management system is a technological response to the challenges of digital transformation in small organizations, such as SMEs, in a strategic and operational way, 
considering that it is a present need of the future entities.

\section{REFERENCES}

[1] Andriolo, A., Battini, D., Grubbström, R., Persona, A. \& Sgarbossa, F. (2014). A century of evolution from Harris's basic lot size model: Survey and research agenda. International Journal of Production Economics, 155, 16-38.

[2] Argüello, G. (2015). Uso de las TIC en las PYME del sector industrial del Área Metropolitana de Bucaramanga. I+D Revista de Investigaciones, 6(2), 125-136.

[3] Asociación Nacional de Instituciones Financieras (2018). Gran Encuesta a las Microempresas. Informe de resultados 2018. Retrieved from: http://www.anif.co/sites/default/ files/publicaciones/gem18_.pdf

[4] Chase, R., Jacobs, R. \& Aquilano, N. (2009). Administración de operaciones. Producción y cadena de suministros. Mexico D. F., Mexico: McGraw-Hill.

[5] Converse, T., Park, J. \& Morgan, C. (2004). PHP5 and MySQL Bible. Indianapolis, United States: Wiley Publishing Inc.

[6] Correa, A. \& Gómez, R. (2009). Tecnologías de la información en la cadena de suministro. DINA, 76(157), 37-48.

[7] Correa, A., Álvarez, C. \& Gómez, R. (2010). Sistemas de identificación por radiofrecuencia, código de barras y su relación con la gestión de la cadena de suministro. Estudios Gerenciales, 26(116), 115-141.

[8] Díaz, F., Harari, I. \& Amadeo, A. (2013). Guía de recomendaciones para diseño de software centrado en el usuario. La Plata, Argentina: Editorial de la Universidad de La Plata.

[9] Duong, L., Wood, L. \& Wang, W. (2015). A Multi-criteria Inventory Management System for Perishable \& Substitutable Products. Procedia Manufacturing, 2, 66-76.

[10] Gauchat, J. (2012). El gran libro de HTML5, CSS3 y Javascript. Barcelona, Spain: Marcombo Ediciones Técnicas.

[11] Gutiérrez, V. \& Rodríguez, L. (2008). Diagnóstico regional de gestión de inventarios en la industria de producción y distribución de bienes. Revista Facultad de Ingenieria Uniersidad de. Antioquía, (45), 157-171.
[12] Hernández, R., Fernández, C. \& Baptista, P. (2014). Metodología de la investigación. Mexico D. F., Mexico: McGraw-Hill.

[13] Hillier, F. \& Lieberman, G. (2010). Introducción a la investigación de operaciones. Mexico D. F., Mexico: McGraw-Hill.

[14] Lind, D., Marchal, W. \& Wathen, S. (2012). Estadística aplicada a los negocios y la economía. Mexico D. F., Mexico: McGraw-Hill.

[15] Morcela, O. \& Nicolao, J. (2011). Programación maestra de la producción con gestión de inventarios de productos terminados para PyMEs. Caso de aplicación: PyME local, sector alimentos congelados. (Undergraduate Thesis). Universidad Nacional de Mar de Plata, La Plata.

[16] Osorio, C. (2008). Modelos para el control de inventarios en las pymes. Panorama, 2(6), 4-10.

[17] Pérez, R., Mosquera, S. \& Bravo, J. (2012). Aplicación de modelos de pronósticos en productos de consumo masivo. Biotecnología en el Sector Agropecuario y Agroindustrial, 10(2), 117-125.

[18] Piña, J. (2012). Determinación de la cantidad económica de pedido en una empresa cauchera venezolana aplicando la técnica LIMIT. Ingeniería Industrial. Actualidad $y$ Nuevas Tendencias, 3(9), 61-72.

[19] Pressman, R. (2010). Ingeniería del software. Un enfoque práctico. Mexico D. F., Mexico: McGraw-Hill.

[20] Render, B. \& Heizer, J. (2004). Principios de administración de operaciones. Mexico D. F., Mexico: Pearson Educación.

[21] Quigley, E., \& Gargenta, M. (2007). PHP and MySQL by example. Massachusetts, United States: Pearson Education Inc.

[22] Quintero, J. (2018). Las pymes en Colombia y las barreras para su desarrollo y perdurabilidad. (Undergraduate Thesis). Universidad Militar Nueva Granada, Bogotá.

[23] Regalado, R. (2007). Las MIPYMES en Latinoamérica. S. I.: Organización Latinoamericana de Administración.

[24] Sandoval, A. (2016). Uso de códigos QR en unidades didácticas. Revista de Posgrado y Sociedad. Sistema de Estudios de Posgrado, 14(1), 39-47. 
[25] Servera, F., Gil, I. \& Fuentes, M. (2009). La influencia de la calidad de servicio logístico en la lealtad: un análisis del papel moderador de las TIC. Investigaciones Europeas de Dirección y Economía de La Empresa, 15(3), 37-58.

[26] Taha, H. (2012). Investigación de Operaciones. Mexico D. F., Mexico: Pearson.
[27] Valderrey, P. (2013). Administración de sistemas gestores de bases de datos. Madrid, Spain: Ecoe Ediciones.

[28] Vila, J. (2011). Los códigos QR aplicados a la educación. Comunicación y pedagogía: Nuevas tecnologías y recursos didácticos, (253), 27-29. 